\title{
Photoelectric, Nonlinear Optical, and Photorefractive Properties of Polyvinylcarbazole Composites with Graphene
}

\author{
A. D. Grishina ${ }^{a}$, T. V. Krivenko ${ }^{a}$, V. V. Savel'ev ${ }^{a}$, R. W. Rychwalski ${ }^{b}$, and A. V. Vannikov ${ }^{a}$ \\ ${ }^{a}$ Frumkin Institute of Physical Chemistry and Electrochemistry, Russian Academy of Sciences, \\ Leninskii pr. 31, Moscow, 119071 Russia \\ e-mail:van@elchem.ac.ru \\ ${ }^{b}$ Department of Materials Science and Manufacturing Technology, Chalmers University of Technology, \\ SE-41296 Göteborg, Sweden
}

Received August 14, 2012; in final form, October 10, 2012

\begin{abstract}
Polyvinylcarbazole (PVK) composites containing graphene in an amount of somewhat less than $0.15 \mathrm{wt} \%$ exhibit third-order dielectric susceptibility due to the presence of graphene, as well as photoelectric and photorefractive sensitivity at $1064 \mathrm{~nm}$. The photorefractive (PR) effect is known to occur in a polymer composite that possesses both photoelectric sensitivity and nonlinear optical properties. The photoelectric, nonlinear optical, and PR properties of PVK composites with graphene have been considered in this paper.
\end{abstract}

DOI: $10.1134 / \mathrm{S} 0018143913020057$

Graphene monolayers were discovered in 2004 [1], and their structure (hexagonally symmetric, covalently bonded 2D carbon monolayers) was established. A graphene monolayer has a thickness of the carbon atom. A.K. Geim, one of the discoverers, notes that being the thinnest, graphene is also the most robust and hardest of known materials. Charge carriers exhibit giant intrinsic mobility in it, have zero effective mass, and move over a few micrometers without scattering at room temperature. Graphene can sustain six orders of magnitude higher current densities than copper, shows a record-breaking thermal conductivity, and is impermeable to gases [2]. Excellent electronic characteristics, heat resistance, chemical stability, transparency, and good mechanical properties have been the impetus for research-and-development works in the line of designing and manufacturing a variety of graphene-based devices [3, 4]. Regarding the photonic and optoelectronic properties of graphene, their extensive study was initiated in 2008 after the advent of the most inexpensive method for liquid-phase exfoliation of graphite [5, 6] by long-term sonication. In the resulting slurry, most individual flakes contain three to four graphene monolayers and a small portion contains up to 15 monolayers [6]. The main focus was on the development of optical limiters, which are used when rapid reduction in transmission with a sharp rise in light intensity is needed. To assess the effect, the openaperture $z$-scan technique was used $[4,7,8]$ (see the Experimental section). During $z$-scanning, the intensity of laser radiation incident on a sample can be increased by five orders of magnitude or more by moving the sample along the focused laser beam. It was shown that such an increase in laser radiation intensity in a graphene suspension or a graphene mixture with oligothiophene leads to only a few percent decrease in optical transmission as a result of twophotonic and twoquantum processes [8]. However, the transmission decreased by $\sim 70 \%$ when the graphene was grafted to oligothiophene [8]. A similar effect was observed after graphene grafting onto polyvinylcarbazole (PVK) [4]. This effect is believed $[4,8]$ to be due to photoinduced energy transfer.

\section{EXPERIMENTAL}

The photoelectric and PR characteristics of the composites were measured using a continuous $\mathrm{Nd}$ : YAG laser with an emission wavelength of $1064 \mathrm{~nm}$. To make the composites, we used the dispersion preparation procedure developed in [9]. Graphene was introduced into tetrachloroethane (TCE) and dispersed for $48 \mathrm{~h}$ in a UZDN-A ultrasonic disperser. After that. the solvent dispersion was allowed to settle, with the undertreated graphene partly precipitating as a black sediment on the bottom of the vessel and partly accumulating on the surface. Then, an appropriate quantity of the dispersion was withdrawn with a micropipette, to which PVK (Aldrich, $M_{W}=1.1 \times 10^{6}$; glass transition temperature, $200^{\circ} \mathrm{C}$ ) was added, and the mixture was subjected to three 30 -min runs of agitation using a magnetic stirrer with heating to $60^{\circ} \mathrm{C}$. Electronic absorption spectra were recorded on a Shimadzu UV-3101PC spectrophotometer in both the graphene/TCE dispersion and PVK-graphene composites supported on a quartz substrate. The composites on the quartz substrate were also used to measure 
the nonlinear optical properties. Photorefractive measurement cells were prepared as follows: first, to reduce the dark current, an $\mathrm{Al}_{2} \mathrm{O}_{3}$ dielectric film was deposited on the electrode, which was a glass substrate coated with a conducting $\mathrm{In}_{2} \mathrm{O}_{3}: \mathrm{SnO}_{2}$ (ITO) film. Then, a solution of the polymer composition was cast on the top and dried at $60^{\circ} \mathrm{C}$ for a sufficiently long time. After evaporation of the solvent, the thickness of the composite was first measured with an interferometer (PR layer thickness was about 6.5-12 $\mu \mathrm{m}$ ). After that, an upper glass plate with an ITO electrode coated with a thin polyvinylalcohol film, capable of adsorbing molecular oxygen, was pressed to the composite. The resulting cell was compressed under a low pressure at $60^{\circ} \mathrm{C}$ for $5 \mathrm{~min}$. Then, the samples were placed in a vacuum chamber and stored before measurement.

Photoelectric measurements were made after measuring the PR characteristics on the same samples. The upper electrode was removed, and an opaque silver paste was applied as the second electrode to have a diameter of about $2.5 \mathrm{~mm}$. The polymer layer was illuminated with a laser beam on the transparent ITO electrode side.

In measuring the PR effect, the laser beam was first split into two equal beams (beams 1 and 2), which were then brought together and crossed in the polymer layer, creating an interference pattern. The bisector of the angle between the incident beams intersected the layer at an angle of $\phi=-45^{\circ}$. The angle between the beams was $2 \theta=15^{\circ}$ [10]. The maximum intensity of beams 1 and 2 at the entrance to the layer was $I_{1}(0)=$ $I_{2}(0)=0.15 \mathrm{~W} / \mathrm{cm}^{2}$. The diameter of each of the beams incident on the layer was about $4 \mathrm{~mm}$ in their intersection region. The spacing of the interference pattern at $\lambda=1064 \mathrm{~nm}$ was $\Lambda=\lambda /\left(2 n_{0} \sin \theta\right)=2.7 \mu \mathrm{m}$, since $\theta=$ $7.5^{\circ}$ and the refractive index of PVK is $n_{0}=1.5$.

To measure third-order nonlinearity, we used a $z$-scan method based on a femtosecond pulse laser [11]. The basic element of the assembling was an Origami-10 pulsed femtosecond laser, which emits radiation with a wavelength of $\lambda=1030 \mathrm{~nm}$. The pulse duration was $217 \mathrm{fs}$. The laser beam was focused by lens 4 with a focal length of $6.5 \mathrm{~cm}$. A movable unit made it possible to move ( $z$-scan) the sample along the laser beam, covering the prefocal $(-z)$, lens focus $(z=$ $0)$, and postfocal $(+z)$ regions. During movement, the sample was stopped after $1 \mathrm{~mm}$ and transmittance was measured. The transmission of light was measured in two modes, $T_{\mathrm{CA}}$ (closed aperture transmittance) with the aperture mounted on the photodetector and $T_{\mathrm{OA}}$ (open aperture transmittance) without an aperture.

The pulse repetition frequency was $74.82 \times 10^{6} \mathrm{~s}^{-1}$. The average power of laser radiation was $0.15 \mathrm{~J} / \mathrm{s}$. Consequently, the energy per pulse at a pulse duration of $217 \mathrm{fs}$ was $I=0.15 /\left(74.82 \times 10^{6} \times 217 \times 10^{-15}\right)=$ $9.24 \times 10^{3} \mathrm{~W}$. Repeated measurements showed that the beam radius at the focus (waist radius) was about $w_{0}=$
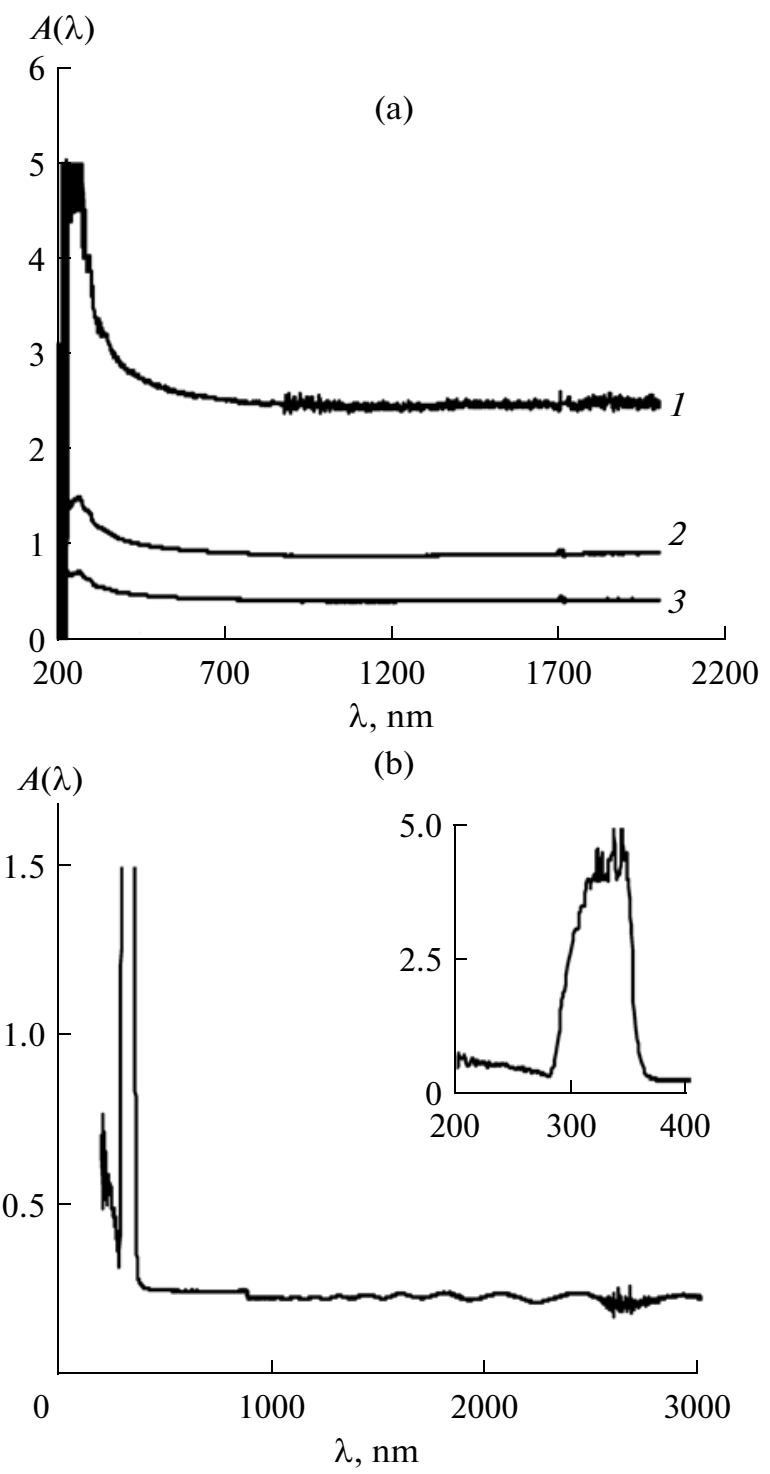

Fig. 1. (a) Optical absorption spectra of a graphene/TCE dispersion with a graphene content of $(1) 1$, (2) 0.4 , or (3) $0.125 \mathrm{mg} / \mathrm{mL}$. (b) Optical absorption of the composite PVK/2.2 wt \% graphene. The inset shows the PVK absorption region.

$20.6 \mu \mathrm{m}$. Therefore, the light intensity at the waist was $I_{0}=I /\left(\pi w_{0}^{2}\right)=6.92 \times 10^{8} \mathrm{~W} / \mathrm{cm}^{2}[11]$.

\section{RESULTS AND DISCUSSION}

Figure 1a shows the optical absorption spectra of the graphene/TCE dispersion, as measured in a cell with a thickness of $0.1 \mathrm{~cm}$. Figure $1 \mathrm{~b}$ shows optical absorption in a PVK/2.2 wt \% graphene layer of a $12 \mu \mathrm{m}$ thickness. The inset of Fig. $1 \mathrm{~b}$ demonstrates the PVK absorption range. Optical absorption of graphene does not change significantly throughout the measurement range of $400 \mathrm{~nm}$ to $3000 \mathrm{~nm}$. The spectrum of 


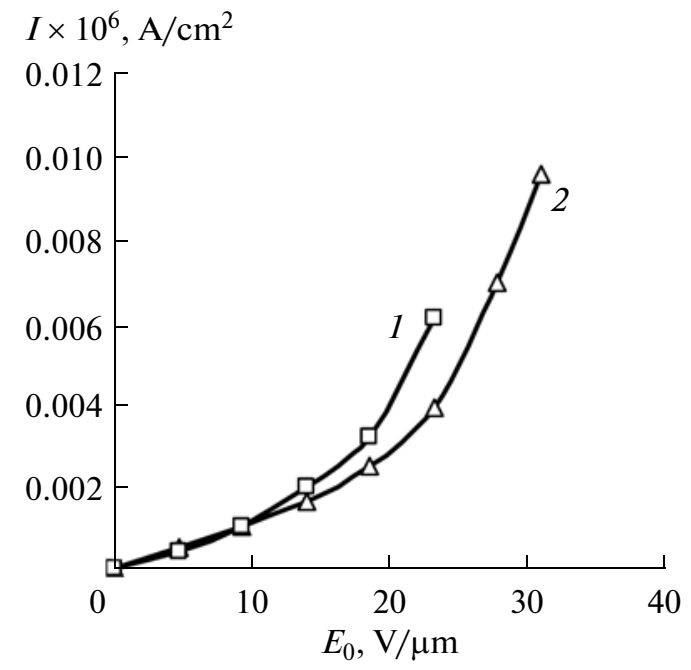

Fig. 2. Field dependences of photocurrent $j_{\mathrm{ph}}$ and dark current $j_{\mathrm{d}}$ in the $\mathrm{PVK} / \leq 0.15 \mathrm{wt} \%$ graphene composite.

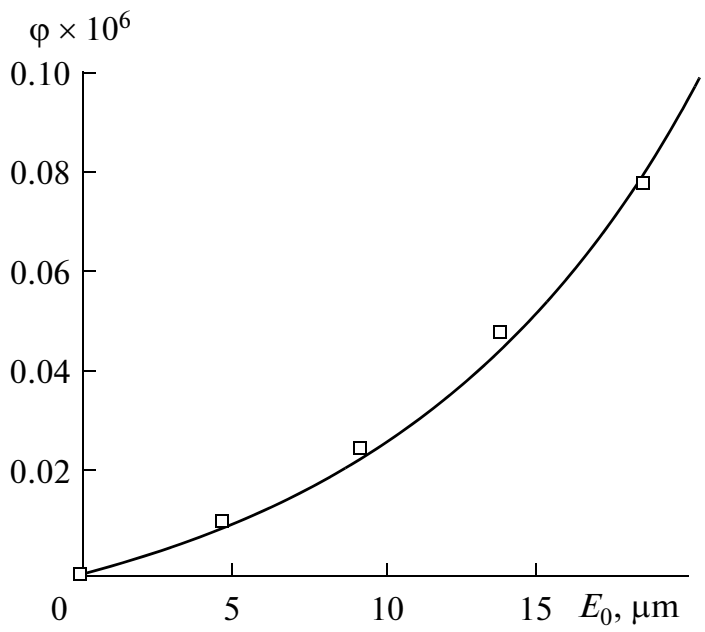

Fig. 3. Dependence of the quantum efficiency $\varphi$ on the applied field $E_{0}$. The solid curve has been constructed on the basis of the Onsager equation for a quantum yield of thermalized electron-hole pairs of $\varphi_{0}=1$ and the initial radius of separation of $r_{0}=9.8 \AA$.

$\varphi_{0} \times P\left(r_{0}, E_{0}\right)$ calculated accurate to $E_{0}^{3}$. Here, $\varphi_{0}$ is the quantum yield of thermalized electron-hole pairs with an initial separation radius $r_{0}$ and $P\left(r_{0}, E_{0}\right)$ is the probability that the charges in the pairs escape recombination with the separation radius $r_{0}$ :

$$
\begin{aligned}
& P\left(r_{0}, E_{0}\right)=\exp \left(-r_{\mathrm{c}} / r_{0}\right)\left\{\left[1+\left(r_{\mathrm{c}} / r_{0}\right)\left(e E_{0} r_{0} / 2 k T\right)-\right.\right. \\
& \left(r_{\mathrm{c}} / r_{0}\right) K_{1}\left(e E_{0} r_{0} / 2 k T\right)^{2}+\left(r_{\mathrm{c}} / r_{0}\right) K_{2}\left(e E_{0} r_{0} / 2 k T\right)^{3},
\end{aligned}
$$

graphene precipitated as a black solid after the dispersion and sedimentation.) Figure 2 depicts field dependence curves for steady-state photocurrent measured when saturation was reached after laser switching on and the dark current in the $\mathrm{PVK} / \leq 0.15 \mathrm{wt} \%$ graphene composites. The photocurrent was determined as the difference between the currents measured under illumination $\left(j_{\mathrm{ph}}+j_{\mathrm{d}}\right)$ and in the dark $j_{\mathrm{d}}: j_{\mathrm{ph}}=\left(j_{\mathrm{ph}}+j_{\mathrm{d}}\right)-$ $j_{\mathrm{d}}$. The field dependence of the quantum efficiency for the formation of mobile charge carriers was estimated from the dependence of photocurrent upon the applied electric field $j_{\mathrm{ph}}(E)$ under continuous operation of the $\mathrm{Nd}$ : YAG laser and was calculated by the formula:

$$
\varphi\left(E_{0}\right)=j_{\mathrm{ph}}\left(E_{0}\right) h v /\left[e \times I_{0}\left(1-\exp \left(-\alpha_{0} d\right)\right)\right] .
$$

At $1064 \mathrm{~nm}, h v=1.165 \mathrm{eV}(h v / e=1.165 \mathrm{~V})$. The quantity $\left(1-\exp \left(-\alpha_{0} d\right)\right)=\left(1-10^{-\mathrm{A}}\right)=(1-$ $\exp (-2.3 A)$ is the proportion of light energy absorbed in the layer. The layer thickness was $d=6.5 \mu \mathrm{m}$. The measured optical absorbance was $A=0.004$. The linear optical absorption coefficient was $\alpha_{0}=2.3 A / d=$ $14.1 \mathrm{~cm}^{-1}$. Figure 3 shows the quantum efficiency as a function of the applied field. The solid curve in Fig. 3 was plotted according to the Onsager equation $\varphi\left(E_{0}\right)=$ where $K_{1}=\left[2-\left(r_{\mathrm{c}} / r_{0}\right)\right] / 3, \quad K_{2}=\left[1-\left(r_{\mathrm{c}} / r_{0}\right)+\right.$ $\left.\left(r_{\mathrm{c}} / r_{0}\right)^{2} / 6\right] / 2$.

The Onsager formalism-based analysis of a wide array of experimental data on carrier photogeneration in doped PVK by Braun [12] showed that the precursors of free charge carriers are relaxed PVK-dopant charge-transfer states with a radius of separation of $r_{0} \leq$ $10 \AA$. In the calculation of the field dependence of the quantum efficiency by the Onsager equation, we took $r_{0}=9.8 \AA$. The quantum yield of thermalized electron-hole pairs estimated according to Eq. (2) is $\varphi_{0}=$ 1 (solid curve in Fig. 3).

The third-order susceptibility was measured in the $\mathrm{PVK} /$ graphene $\leq 0.15 \mathrm{wt} \%$ composite by the $z$-scan method with a femtosecond laser. As noted above, the laser pulse had a duration of $217 \mathrm{fs}$ and, as such, could not affect the triplet transitions, since it was more than three orders of magnitude shorter than the time of singlet-triplet intersystem crossing. Therefore, the optical nonlinearity is most likely due to twophotonic $S_{0} \rightarrow S_{2}$ absorption.

During $z$-scanning [13] in this study, the transmission of light was measured in the two modes, with the aperture mounted on the photodetector $\left(T_{\mathrm{CA}}\right)$ and without the aperture ( $T_{\mathrm{OA}}$ mode). At high light inten- 


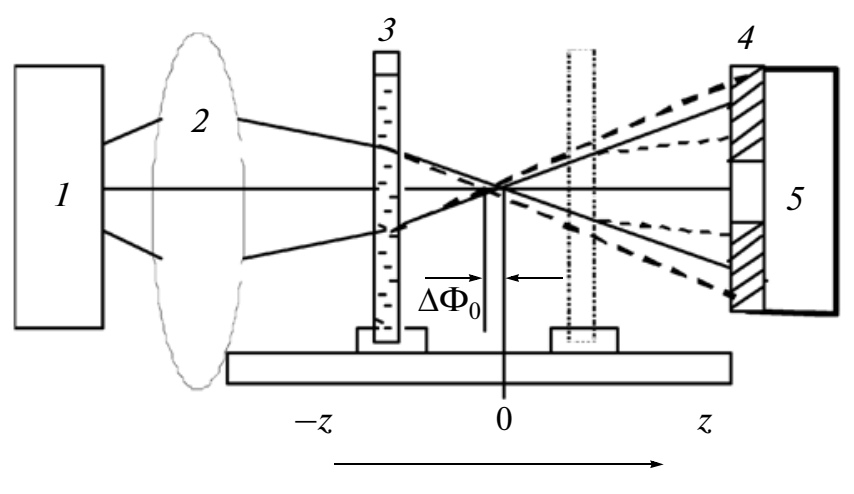

Fig. 4. Z-scan setup: (1) 1030-nm femtosecond laser, (2) focusing lens, (3) movable sample, (4) 1-mm hole aperture, and (5) photodetector.

sity in the focal region, the space polarization of the sample acquires a significant contribution of nonlinear components and the refractive index becomes

$$
n=n_{0}+n_{2} I_{0} .
$$

During $z$-scanning, the refractive index $n$ increases as the sample approaches the focal point with an increase in $I_{0}$ at positive $n_{2}$ and, since the sample acts as an additional focusing lens, the distance to the focus is reduced by the value of phase shift $\Delta \Phi_{0}$. Therefore, the beam diameter in the aperture region increases, resulting in a decrease in the proportion of light entering the aperture hole. In the postfocal region (for positive values of $z$ ), the increase in the refractive index at high intensities $I_{0}$ leads to a decrease in the beam diameter on the aperture and, hence, an increase in the proportion of light entering the aperture hole. Therefore, the photodetector records $z$-scan $T_{\mathrm{CA}}$ curves with a minimum and a maximum in the prefocal and the postfocal region, respectively.

Figure 5a presents $T_{\mathrm{CA}}$ as a function of the distance from the focal point $(z=0)$ in the solid PVKgraphene composite. There is a coincidence of transmittance measured 40 and $450 \mu$ s after laser beam opening; i.e., sample heating is not manifested noticeably under these conditions. The experimental data points were approximated by the theoretical relationship [13]

$$
T_{\mathrm{CA}}=1-4 \Delta \Phi_{0} x /\left[\left(x^{2}+1\right)\left(x^{2}+9\right)\right]
$$

via fitting the phase shift $\Delta \Phi_{0}$, which is related to $n_{2}$ by the equation:

$$
n_{2} I_{0}=\Delta \Phi_{0} \lambda / 2 \pi L_{\text {eff }}
$$

Here, $x=z / z_{0}$ is the relative distance from the cell to the focus, $z_{0}=n_{0} \pi w_{0}^{2} / \lambda, L_{\text {eff }}=\left(1-\mathrm{e}^{-\alpha} 0^{L}\right) / \alpha_{0}[13], L=$ $6.5 \mathrm{~mm}$ is the thickness of the composite, and $\alpha_{0}=$ $2.3 \mathrm{~A} / \mathrm{d}=14.1 \mathrm{~cm}^{-1}$ is the optical absorption coefficient at $1030 \mathrm{~nm}$. Hence, $L_{\text {eff }}=0.000645 \mathrm{~cm}$.

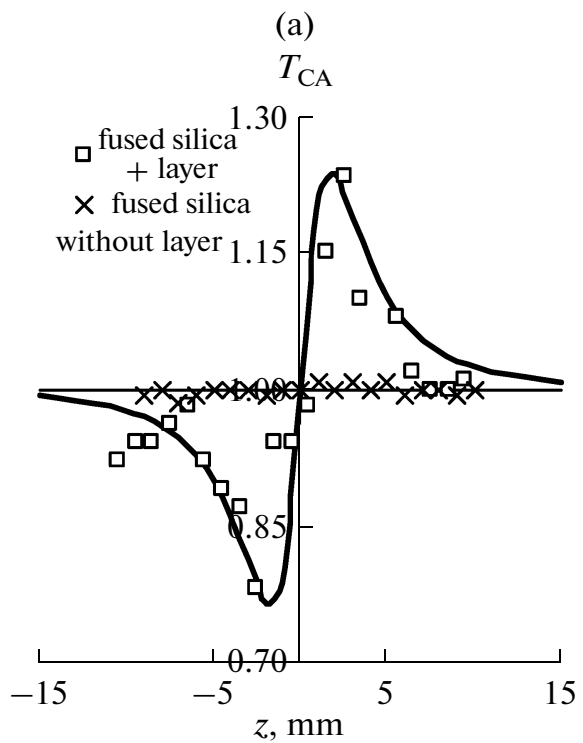

(b)

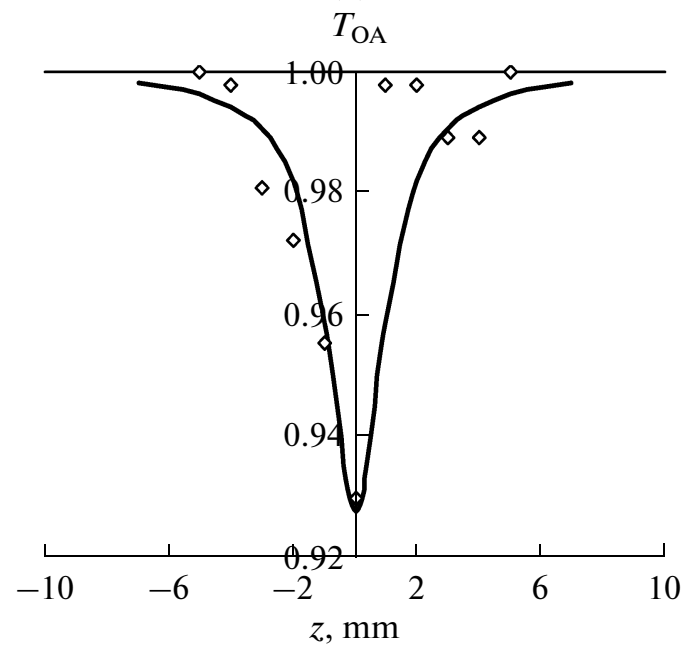

Fig. 5. Dependence of (a) $T_{\mathrm{CA}}$ and (b) $T_{\mathrm{OA}}$ on the distance from the focus $(z=0)$ in the solid PVK $-\leq 0.15 \mathrm{wt} \%$ graphene composite (symbols $\times$ mark $T_{\mathrm{CA}}$ of the fused-silica substrate.) The data points refer to the experimental results and the curves refer to their theoretical approximation by Eq. (4) for $T_{\mathrm{CA}}$ and Eq. (6) for $T_{\mathrm{OA}}$.

The curve in Fig. 5a illustrates Eq. (4) at $\Delta \Phi_{0}=1.2$. From Eq. (5) it follows that $n_{2} I_{0}=0.02$ and $n_{2}=3.2 \times$ $10^{-11}$. The real part of third-order susceptibility is related to $n_{2}$ by $\chi^{(3)}=n_{2} \times\left(n_{0}^{2} / 0.0394\right)$ esu (electrostatic units). Consequently, the real term of susceptibility in the PVK composite with $\leq 0.15 \mathrm{wt} \%$ graphene is $\chi^{(3)}=1.8 \times 10^{-9}$ esu.

The imaginary part of the susceptibility is determined by nonlinear optical absorption $\beta$. At high laser intensities, the optical absorption coefficient includes the linear $\alpha_{0}$ and nonlinear $\beta I_{0}$ terms: $\alpha=\alpha_{0}+\beta I_{0}$. 


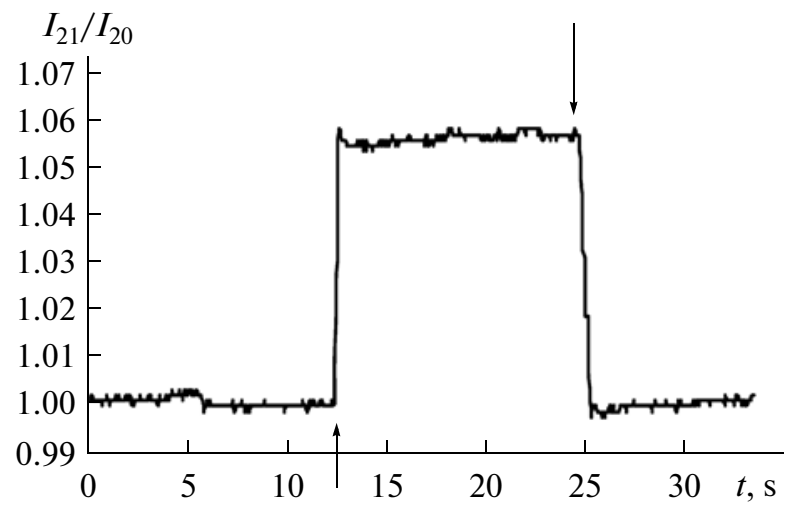

Fig. 6. Change in the intensity of beam 2 by turning beam 1 on $(\uparrow)$ and off $(\downarrow)$ in the PVK/0.8 wt\% grapheme composite. $I_{21}$ and $I_{20}$-are the-intensities of beam 2 with beam 1 switched on and off, respectively. The picture does not vary when an electric field is applied or its polarity changes.

The quantity $\beta$ is evaluated from the open aperture transmittance using the following equation [14]

$$
T_{\mathrm{OA}}=\ln \left(1+q_{0} /\left(1+x^{2}\right)\right) /\left(q_{0} /\left(1+x^{2}\right)\right),
$$

where $q_{0}=\beta I_{0} L_{\text {eff }}$. Approximation of the experimental data $\mathrm{s}$ in Fig. $5 \mathrm{~b}$ by Eq. gives $q_{0}=0.16$. Consequently, $\beta=0.16 / I_{0} L_{\text {eff }}=2.6 \times 10^{-7} \mathrm{~cm} / \mathrm{W}$ and the imaginary part of susceptibility is $\chi^{(3)}=(\beta \lambda / 4 \pi) \times\left(n_{0}^{2} / 0.0394\right)=$ $1.2 \times 10^{-10}$ esu. Since the real part of susceptibility is much greater than the imaginary part, the net susceptibility, $\left[\left(\operatorname{Re} \chi^{(3)}\right)^{2}+\left(\operatorname{Im} \chi^{(3)}\right)^{2}\right]^{0.5}=1.82 \times 10^{-9} \mathrm{esu}$, is determined by the real part of the susceptibility of the sample.

As seen in Fig. 5b, optical transmittance of the layer is reduced by $7 \%$ with an increase in laser radiation intensity $I_{0}$ by about five orders of magnitude (to a value of $6.9 \times 10^{8} \mathrm{~W} / \mathrm{cm}^{2}$ ).

The PR effect was measured at a laser wavelength of $1064 \mathrm{~nm}$. The PR effect appears in a photosensitive polymer composite by the interference of two coherent laser beams, in which electron-hole pairs are generated in the bright fringes. Under the influence of a constant electric field $E_{0}$ applied to the sample, the charges separate and drift in the opposite directions until their capture in deep traps. The trapped charges of opposite signs form a periodic electric field of space charge $E_{\mathrm{sc}}$ (subscript sc stands for space charge). The field $\left(E_{0}+E_{\mathrm{sc}}\right)$ polarizes nonlinear optical chromophores (graphene), thereby causing periodic refractive index modulation $\Delta n=\left(2 \pi / n_{0}\right) \chi^{(3)}\left(E_{0}+\right.$ $\left.E_{\mathrm{sc}}\right)^{2}$, i.e., providing for generation of a phase grating in the polymer layer.

The PR effect does not occur unless electrons and holes before trapping are displaced to different distances from the site of generation. In our experiment, as will be shown below, the intensity of beam 2 increased when a negative potential was applied to the electrode at the exit of laser beams. Hence, it follows that holes are more mobile than electrons in the $\mathrm{PVK} /$ graphene composite. It may be assumed that the following reaction occurs after photoexcitation of graphene at $1064 \mathrm{~nm}$ :

$$
\begin{gathered}
\text { Graphene }+h v \rightarrow \text { Graphene }{ }^{*} ; \\
\text { Graphene }^{*}+\mathrm{PVK} \rightarrow \text { Graphene }^{-}+\mathrm{PVK}^{+} \\
\text {and subsequent transport of holes } \rightarrow \mathrm{PVK}^{+}+ \\
\text {PVK } \rightarrow \text { PVK }+\mathrm{PVK}^{+\cdot} \text { etc. }
\end{gathered}
$$

Owing to the low concentration of graphene, $\leq 0.15 \mathrm{wt} \%$, its single particles do not interact with each other and there is no electron transport.

When a negative potential is applied to the output electrode, the diffraction grating is displaced with respect to the interference pattern by a distance $(-\Delta x)$ or a phase $-\psi=2 \pi \Delta x / \Lambda$, where $\Lambda$ is the period of the grating. Beam 2, directed from the fringe to the grating, coincides in both direction and phase with the part of pump beam 1 reflected from the grating, and their constructive interference provides amplification of the transmitted beam 2. Hence, beam 2 is an object beam. Pump beam 1 is in antiphase with the reflected part of object beam 2, and their destructive interference leads to attenuation of the pump beam.

Below, it will be shown that the PR effect was observed only at a low graphene concentration of $\leq 0.15 \mathrm{wt} \%$. With a higher graphene content of $0.8 \mathrm{wt} \%$ as shown in Fig. 6, the intensity of light $I_{21}$ incident on the photodetector, which is designed for measuring the intensity of beam 2 and, as noted above, offset from the direction of beam 1 by $15^{\circ}$, increases by almost $6 \%$ when beam 1 is switched on. The picture shown in Fig. 6 is preserved in the applied electric field and is independent of its direction. It follows that the $6 \%$ intensity rise in the channel of beam 2 as shown in Fig. 6 is due to scattering of beam 1. (Beam 2 is scattered to the same extent.) The absolute absence of the PR effect in the case of applied electric field is indicates the complete loss of coherence of the beams as a result of scattering.

Figure 7 shows two-beam coupling kinetic curves measured in the $\mathrm{PVK} / \leq 0.15 \mathrm{wt} \%$ graphene composite at a laser intensity of $I_{1}(0)=I_{2}(0)=0.15 \mathrm{~W} / \mathrm{cm}^{2}$ in a field of $E_{0}=111 \mathrm{~V} / \mu \mathrm{m}$ (Figs. $7 \mathrm{a}$ and $7 \mathrm{~b}$ ) and $133 \mathrm{~W} / \mathrm{cm}^{2}$ (Fig. 7c). Measured was the intensity of the beam 2 transmitted through the layer when beam 1 was switched on $\left(1\left(I_{21}\right)\right.$ and in its absence $\left(I_{20}\right)$. The measurements were made as follows. First, beam 2 and the electric field $E_{0}$ were successively turned on, and, a few seconds later, beam 1 was turned on (upwards arrows) and then off (downward arrows). As shown in Figs. $7 \mathrm{a}$ and $7 \mathrm{~b}$, the change in intensity in the channel of beam 2 with beam 1 switched on is composed of two contributions, a sharp increase in intensity by $\sim 1.1 \%$ in both figures is due to the fact that scattered beam 1 gets into the channel of beam 2. A comparison of Figs. 6 and 7 shows that scattering is reduced from 6 to 
$1.1 \%$ in proportion to grapheme concentration as the graphene content decreases from 0.8 to $0.15 \%$.

The PR effect is manifested in an increase in intensity of beam 2 by $\Delta$ when the electric field $E_{0}$ is directed from the input to the output electrode (Fig. 7a). When the direction of the electric field is reversed (Fig. 7b), the intensity of beam 2 decreased by the same value of $\Delta$. These changes are due to the fact that in the former case, as noted above, the grating was displaced relative to the interference pattern by the phase $\psi=-2 \pi \Delta x / \Lambda$ and beam 2 and 1 were the object and pump beams, respectively. In the latter case, the grating was displaced relative to interference pattern by the phase $\psi=+2 \pi \Delta x / \Lambda$, beam 1 became the object beam, and beam 2 became the pump beam. Such a change in the function of the beams with a change of the direction of the applied electric field is unequivocal evidence for the PR effect.

The gain factor is $g_{0}=1+\Delta=1.0043$ at $111 \mathrm{~V} / \mu \mathrm{m}$ (Fig. 7a). The appearance of the PR effect at a graphene content of $\leq 0.15 \mathrm{wt} \%$ and $1.1 \%$ scattering means that some portions of beams 1 and 2 retain coherence under these conditions.

From the data in Fig. 7a and 7b, it is seen that the PR part of the $I_{21} / I_{20}$ ratio first reaches a maximum and then gradually decreases. (In Fig. 7b, it first reaches a minimum and then starts increasing.) These effects are associated with gradual buildup of space charge in the near-electrode area during passing dark current and with a corresponding reduction of the field $E_{0}$ in the sample [15]. To remove the relevant space charge, kinetic curves were recorded with alternating polarity of the electrodes.

Only the PR gain of beam 2 is given in Fig. 7c. The rise in the $I_{21} / I_{20}$ ratio fits well with the equation

$$
I_{21} / I_{20}=1+\left(g_{0}-1\right)\left\{1-\exp \left[-\left(t-t_{0}\right) / \tau\right]\right\} .
$$

Here, $\tau$ is the response time (grating formation time constant). The growth in the $I_{21} / I_{20}$ ratio shown in Fig. $7 \mathrm{c}$ is consistent with Eq. (7) at $g_{0}=1.012$ and $\tau=$ $0.2 \mathrm{~s}$.

The two-beam gain coefficient $\Gamma$ measured at equal intensities of the input beams $I_{1}(0)=I_{2}(0)$ was calculated by the equation

$$
\Gamma=\mathrm{LN}\left(g_{0} /\left(2-g_{0}\right)\right) / L \text {. }
$$

Here $L=d / \cos (\phi-\theta)$ is the optical path length of beam 2. The two-beam gain coefficients are $\Gamma=$ $7.6 \mathrm{~cm}^{-1}$ at $E_{0}=111 \mathrm{~V} / \mu \mathrm{m}$ and $\Gamma=21.2 \mathrm{~cm}^{-1}$ at $E_{0}=$ $133 \mathrm{~V} / \mu \mathrm{m}$. Since the optical absorption coefficient in the composite is $\alpha_{0}=14.1 \mathrm{~cm}^{-1}$ as noted above, the net gain has a positive value only at $133 \mathrm{~V} / \mu \mathrm{m}$ and is $\Gamma-\alpha_{0}=7.1 \mathrm{~cm}^{-1}$. Such low values for the two-beam gain coefficients are due to scattering of beams 1 and 2 , leading to the loss of their coherence, the necessary condition for constructive interference.

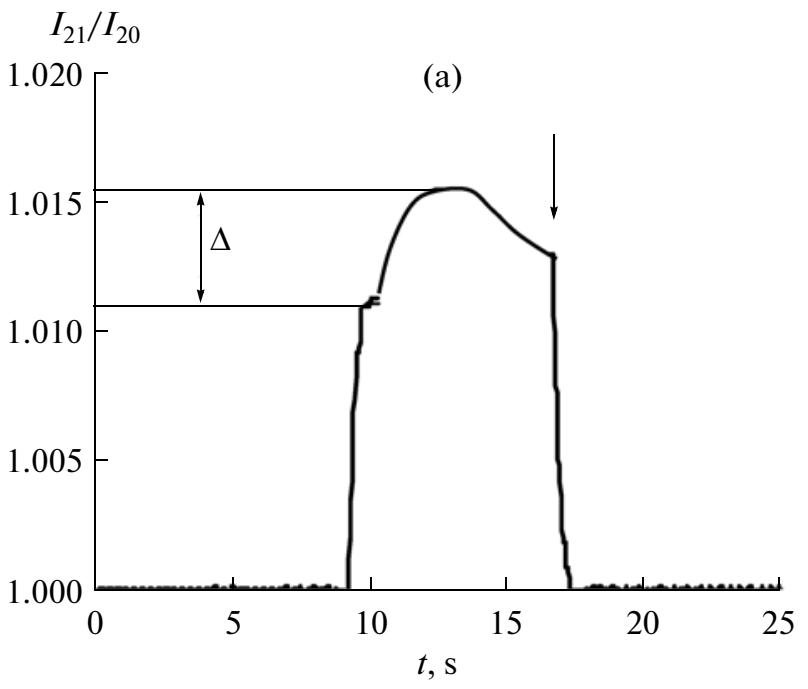

(b)

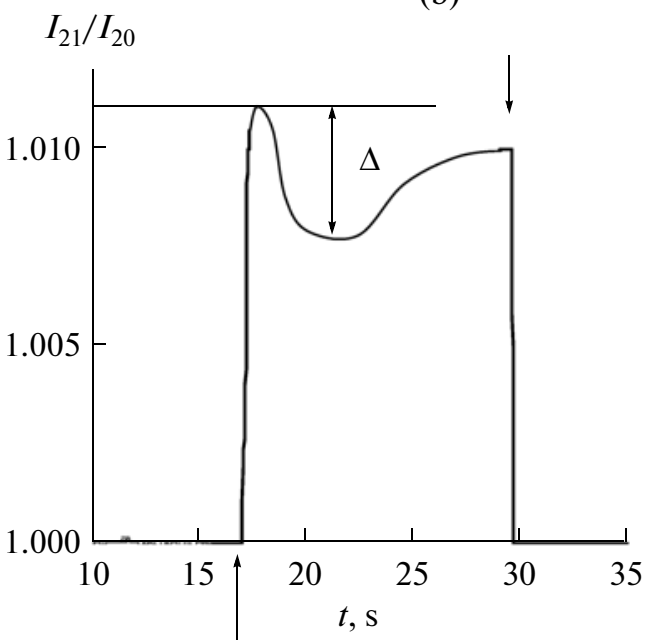

(c)

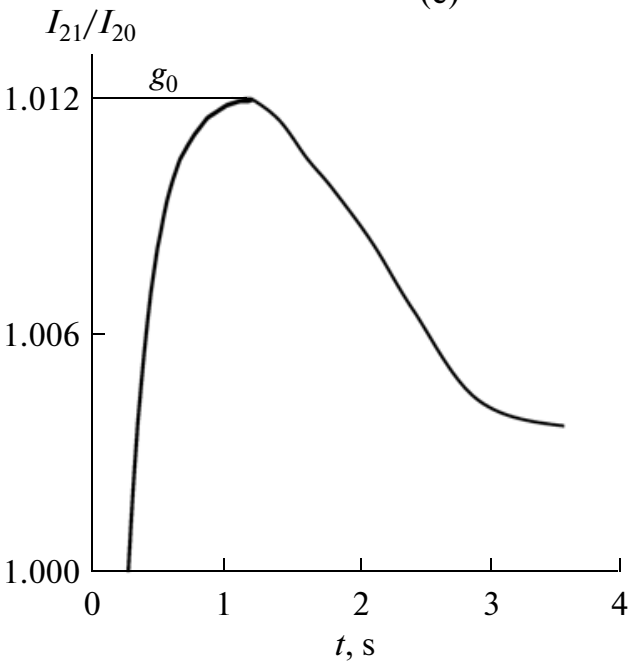

Fig. 7. Change in the intensity of the beam 2 by turning beam 1 on $(\uparrow)$ and off $(\downarrow)$ in the $\leq 0.15$ wt\% $\mathrm{PVK} /$ graphene composite. The applied field is $(\mathrm{a}, \mathrm{b})$ $111 \mathrm{~V} / \mathrm{m}$ or (c) $133 \mathrm{~V} / \mu \mathrm{m}$. The field $E_{0}$ is directed from the electrode at the beam entrance $(\mathrm{a}, \mathrm{c})$ to the output electrode and (b) in the opposite direction. 


\section{CONCLUSIONS}

Graphene in a small amount of somewhat less than 0.15 wt \% admixed to PVK imparts photoelectric and PR sensitivity at $1064 \mathrm{~nm}$ to the PVK/graphene composite, as well as third-order dielectric susceptibility. The quantum yield of thermalized electron-hole pairs determined with the use of the Onsager equation is $\varphi_{0}=1$ with the initial pair separation radius of $r_{0}=$ $9.8 \AA$.

It has been found that the third-order dielectric susceptibility in this composite is $\chi^{(3)}=1.8 \times 10^{-9}$ esu.

The PR gain coefficient of the object laser beam is low, $\Gamma=21.2 \mathrm{~cm}^{-1}$ at an applied field of $E_{0}=$ $133 \mathrm{~V} / \mu \mathrm{m}$. Such a low value of the PR gain coefficient is due to the revealed considerable scattering of laser beams by the medium, resulting in the loss of their coherence.

\section{ACKNOWLEDGMENTS}

This work was supported by the Russian Foundation for Basic Research, project no. 11-03-00260, and the Swedish Foundation for Strategic Research (SSF).

\section{REFERENCES}

1. Novoselov, K.S., Geim, A.K., Morozov, S.V., Jiang, D., Zhang, Y., Dubonos, S.V., Grigorieva, I.V., and Firsov, A.A., Science, 2004, vol. 306 (5696), p. 666.

2. Geim, A.K., Science, 2009, vol. 324 (5934), p. 1530.

3. Blake, P., Brimicombe, P.D., Nair, R.R., Booth, T.J., Jang, D., Schedin, F., Ponomarenko, L.A., Morozov, S.V., Gleeson, H.F., Hill, E.W., Geim, A.K., and Novoselov, K.S., Nano Letters, 2008, vol. 8, no. 6, p. 1704.

4. Wang, J., Chen, Y., Li, R., Dong, H., Zhang, L., Lotya, M., Coleman, J.N., and Blau, W.J., in Carbon Nanotubes-Synthesis, Characterization, Applications, S. Yellampalli, Ed., InTech, 2011, p. 397.

5. Hernandez, Y., Nicolosi, V., Lotya, M., and Blighe, F.M., Nat. Nanotechnol., 2008, vol. 3, no. 9, p. 563.

6. Lotya, M., Hernandez, Y., King, P.J., and Smith, R.J., J. Am. Chem. Soc., 2009, vol. 131, p. 3611.

7. Liu, Z., Wang, Y., Zhang, X., Xu, Y., Chen, Y., and Tian, J., Appl. Phys. Lett., 2009, vol. 94, p. 021902.

8. Zhang, X.L., Zhao, X., Liu, Z.B., Liu, Y.S., Chen, Y.S., and Tian, J.G., Opt. Express, 2009, vol. 17, no. 26, p. 23959.

9. Persson, H., Yao, Y., Klement, U., and Rychwalski, R.W., Express Polym. Lett., 2012, vol. 6, no. 2, p. 142.

10. Vannikov, A.V. and Grishina, A.D., High Energy Chem., 2007, vol. 41, no. 3, p. 162.

11. Laryushkin, A.S., Savel'ev, V.V., Zolotarevskii, V.I., Grishina, A.D., Krivenko, T.V., Rychwalski, R.W., and Vannikov, A.V., High Energy Chem., 2011, vol. 45, no. 3, p. 245.

12. Braun, C.L., J. Chem. Phys., 1984, vol. 80, no. 9, p. 4157.

13. Sheik-Bahae, M., Said, A.A., Wei, T-H., Hagan, D.J., and van Stryland, E.W., IEEE J. Quantum Electron., 1990, vol. 26, no. 4, p. 760.

14. Sutherland, R.L., Handbook of Nonlinear Optics, New York: Marcel Dekker, 1996.

15. Borsenberger, P.M. and Weiss, D.S., Organic Photoreceptors for Xerography, New York: Marcel Dekker, 1998. 